\title{
ECONOMIC IMPLICATIONS OF SMARTPHONE APPLICATIONS PROMOTING THE PHYSICAL ACTIVITY
}

\author{
Felician ALECU \\ Bucharest University of Economic Studies \\ felician.alecu@ie.ase.ro \\ Paul POCATILU \\ Bucharest University of Economic Studies \\ ppaul@ase.ro \\ Sergiu CAPISIZU \\ Romanian Bar Association \\ sergiu.capisizu@yahoo.com
}

\begin{abstract}
Today we are experiencing a quick and huge development of the so-called modern fitness movement directly linked to a longer life due to the fact the physical activity is easily able to add years to life, the main benefits being related to getting fit, happiness, physical and mental health improvements, psychological wellbeing, sense of satisfaction, self-esteem, better productivity and performance, all these also having a significant economic impact. Exercise is a very affordable miracle cure generating significant direct health care savings in terms of clinics, doctors, medication and research. The physical activity increase for an active living is an important investment for the future of any society. Applications are quickly moving the market of tracking gadgets from proprietary devices to mobile phones. Various sensors incorporated in the vast majority of the mobile devices in use today are a very cost-effective solution to track the physical effort on a daily basis. The physical activity relative price is an important tool for shaping the public health policies to be implemented by any government.
\end{abstract}

Keywords: smartphone, economic impact, physical activities, sensors, IoT

JEL classification: L86, C88, Y80

DOI: $10.24818 / \mathrm{ie} 2020.01 .04$

\section{Introduction}

Throughout history, physical activity was one of the most important aspects of the human life, including the agricultural activities, maintenance or exhausting manual work, all these involving different degrees of physical effort to be invested into. The amount of this kind of physical work was greatly reduced or even lost in modernity by the technological advances of our days, both at home and at work.

While the activity levels are continuously falling year by year, sports and exercise become a way of life, since the humans were made by design to be active beings. Regular exercise is definitely linked to a longer life, physical activity being commonly considered as a very healthy habit able to easily add years to life.

A few of the main advantages of regular physical activities are related to being fit, happiness, physical and mental health improvements, psychological wellbeing, sense of satisfaction, selfesteem, better productivity and performance.

Today, it is well considered the physical activity can prevent a wide range of lifestyle related diseases, such as the metabolic or cardiovascular disorders linked to unhealthy habits like the improper diet, inactivity or sedentary behavior, all these having a significant economic impact. 
www.conferenceie.ase.ro

Thus, there is no doubt about the fact the physical activity plays an important preventive role against some forms of the most dangerous chronic diseases, inactivity being considered as one of the most important factors for all the early global deaths [1], [2]. Inactivity or insufficient activity is generating a higher occurrence risk for more than 20 health issues including diabetes, cancer, obesity or cardiovascular disease.

The miracle cure named exercise is the all the time most cost-effective medication - no prescription needed, easy to start, very few side effects with a great number of health-related benefits by lowering risks for a wide range of diseases.

The paper is structured as follows. The next section presents the way the technology contributes to a healthier life. The third section focus on the economic consequences of physical activity and inactivity. Section 4 is dealing with the prices associated with physical activity and inactivity. The paper ends with conclusions and future work.

\section{How technology can contribute to a healthier life?}

According to the World Health Organization, around of $25 \%$ of adults are insufficiently active [3] while the obesity simply doubled in the last 50 years as an effect of a fast decline in physical activity rates.

Smartphones can be valuable tools to improve people health and fitness level by the use of applications fighting against sedentary patterns by promoting physical activity and recording different metrics with the help of geolocation, accelerometer, pulse and other sensors, diary, microphone or camera. Other wearables and measuring devices can be wirelessly attached, so the applications can also monitor data coming from various sensors like heart rate, blood pressure, wristband, belt, shoe or smart clothes powered by sensing technologies. By simply sharing the results on the social networks, the social influence of this positive behavioral change can be emphasized with the support coming from friends encouraging such healthier life approaches. Also, rewards could be a very effective way to increase the physical activity level of any individual.

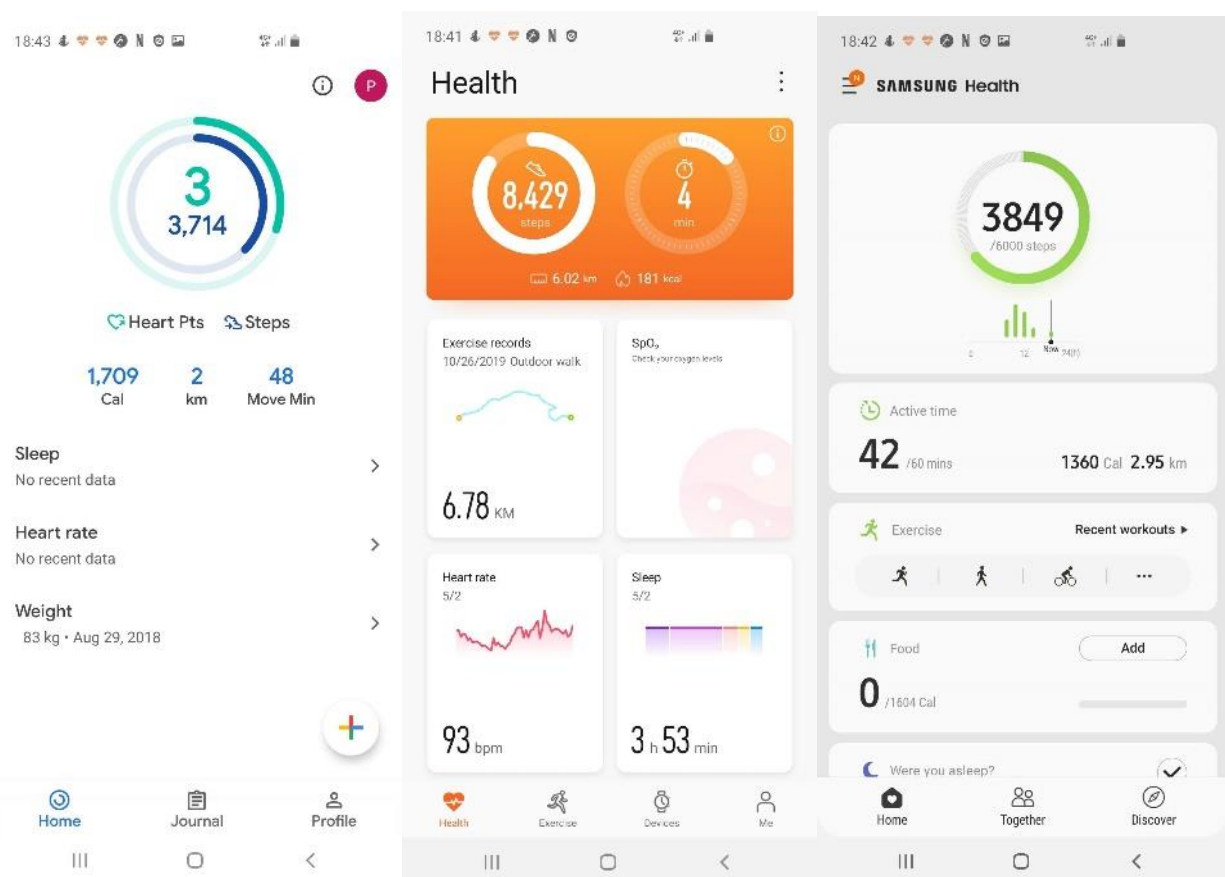

Figure 1. Examples of health-tracking applications: Google Fit, Huawei Health and Samsung Health 
Such applications are helpful for a wide range of health-related goals, like weight loss, stress relief, improved fitness level, better sleep, diet, nutrition or relaxation. Also, applications could be very useful tools to quickly deliver an early warning sign regarding the health condition of the user. Figure 1 presents three examples of such type of applications.

With the help of the applications, behavioral theory-based strategies can be easily applied by simply analyzing and summarizing the data collected during exercises, the applications being able to

- classify the activity type (static, walking, running, stair use, cycling, swimming and so on);

- set daily goals;

- monitor and give feedback about the progress;

- report back the results;

- track overtime;

- provide a customized coaching;

- bring supplementary motivation by the social media sharing of the achievements.

Applications like Apple Health, Google Fit, Huawei Health and Samsung Health are already installed on their own developed devices or can be easily installed on other devices. Table 1 shows a comparison of several health-tracking applications. All the apps requires an account to keep track of data. Most of these applications includes support for activity tracking, goals and targets, social network connections, sleep monitoring.

Table 1. Examples of physical activity applications

\begin{tabular}{|l|l|l|l|}
\hline Application & Platform & $\begin{array}{l}\text { Premium } \\
\text { plans }\end{array}$ & Dedicated for \\
\hline adidas Runtastic & Android, iOS & Yes & $\begin{array}{l}\text { Training } \\
\text { running }\end{array}$ \\
\hline Apple Health & iOS & & \\
\hline Endomondo & Android, iOS & Yes & \\
\hline Google Fit & Android, iOS & & \\
\hline Huawei Health & Android, iOS & & \\
\hline Nike Training Club & Android, iOS & Yes & \\
\hline Samsung Health & Android, iOS & & \\
\hline Strava & Android, iOS & Yes & Running, cycling \\
\hline
\end{tabular}

Wearables are a common choice of our days and there are many types of devices on the market. Figure 2 presents an example of such devices. Usually, these wearable devices are paired with a companion application running on a mobile device, like Apple Heatlh, Huwaei Health, Fitbit, Polar, Samsung, Xiaomi Mi Fit etc.

The decision to buy such a device by individuals should be sufficiently motivating to pay for it. For this reason, the applications are considered the most attractive solution to increase the physical activity level with a magnitude expected to generate long-term benefits in terms of health like reducing risks for cardiovascular diseases or diabetes.

As like in [4], devices with these capabilities, based on IoT and mobile applications, can be developed in order to achieve specific needs. 


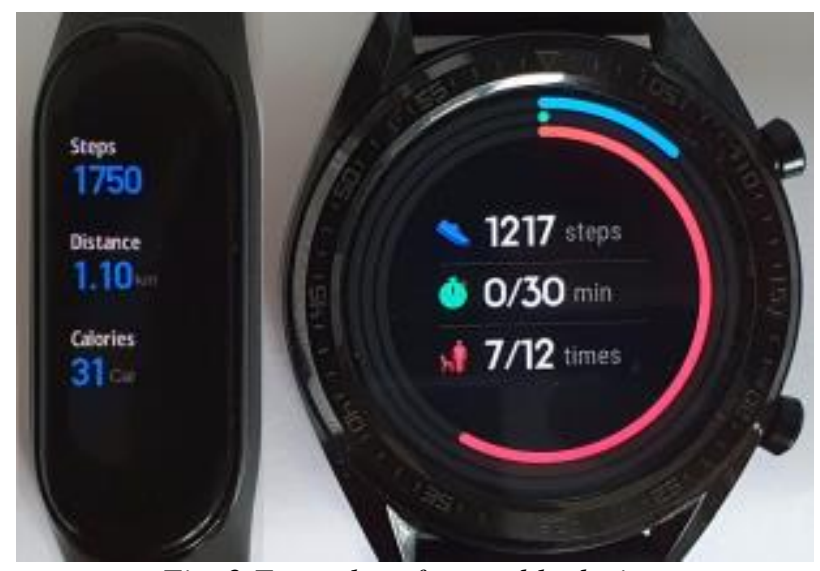

Fig. 2 Examples of wearable devices

Another important benefit of using the applications is the fact no additional device is required to be carried on since persons generally hold the mobile phones with them all the time, so people accessibility to such a device is rather a norm than an exception in the light of the exponential growth of the global smartphone use and their huge penetration rate throughout society.

Physical activity applications, as an inexpensive, user-friendly and accessible solution for almost everyone, will surely become in the future more and more important in promoting workouts and exercises by encouraging the behavioral changes of individuals.

While the humans are considered to be social animals, the community dimensions of any type of physical activity that make the individuals to feel as being part of a group will actually generate a positive attitude that can be enhanced by the use of technology - virtual groups sharing common interests.

Since people usually spend one third of the adult life at work, the employer support to create a so called culture of health is very important by combining personal goals with organizational objectives and management commitment. To sustain an increased level of activity, mobile devices and technology are considered an effective solution to track progress and verify the performed activities in terms of quantity and quality, since the employee engagement rate in such corporate programs is usually low.

Scientific accuracy and short-term efficacy of mobile phone applications is still debatable, but studies are constantly showing [5] the smartphone-recorded figures are only slightly different from the observed or accurate measured values taken with dedicated professional medical devices. So, from a money point of view, it is far more affordable to simply download an application than to invest in an expensive wearable tracker or fitness device.

There is a very few information available about the validity of the data logged by the smartphone applications or wearable trackers since the sensors of the mobile devices are rarely tested for accuracy related to the physical activity records. Some studies, like [6], are showing reliable measures for distance while the energy expenditure estimation was low or even not very accurate at all compared with results coming from the medical devices.

Technology can help to integrate physical activity into the health care standard by recommending the proper exercises to individuals based on the health records.

Advances in artificial intelligence allows applications to better understand people behavior and respond to various questions and issues coming from users.

Another option is to develop dedicated applications using the application programming interfaces (API) provided by the platforms or third parties software development kits (SDK). For example, Android platform includes Activity Recognition API [7] and iOS provides Core Motion framework [8]. A short comparison of these basic APIs is provided in Table 2. In order 
to provide high-level information (such as activity type), machine learning and artificial intelligence models are used.

Table 2. Android and iOS API for activity tracking

\begin{tabular}{|l|l|l|}
\hline API & Availability & Features \\
\hline Activity Recognition API & Android & $\begin{array}{l}\text { Activity type detection } \\
\text { (walk, run etc.) } \\
\text { Activity transitions }\end{array}$ \\
\hline Core Motion framework & iOS & $\begin{array}{l}\text { Activity type detection } \\
\text { (walk, run etc.) } \\
\text { Movement disorder } \\
\text { Read sensors data } \\
\text { (accelerometer, pedometer, } \\
\text { gyroscope etc.) }\end{array}$ \\
\hline
\end{tabular}

Another option is to use system-integrated health application (Google Fit for Android and Apple Health). Android platforms provides Google Fit SDK [9] and on iOS devices, Apple HealthKit [10] is available. Other physical activities applications provide SDK for third party applications.

\section{Economic consequences of physical activity and inactivity}

First, the cost linked to the fitness related activities, such as equipment, wear or participation expenses, represents a big contributor to the global economy. This is considered a negative cost for the individuals performing physical workouts, along with possible injuries, time away from work, participation taxes or long-term wear of the body joints.

On the other hand, an active population generates significant direct health care savings (in terms of clinics, doctors, medication and research) having a positive impact across the overall economy development level. Health care costs per capita may go to half for the persons being considered as physically active. Moreover, if every person would become physically active, these costs may be halved again. In this light, inactivity becomes a critical public health challenge since the technology gradually removed the need of the physical effort concerning many or even the most of our daily tasks.

Apart of these, the indirect inactivity costs related to mood and anxiety disorders are also affecting the global economy by reduced levels of productivity. In average, performing 20 minutes of daily simple and inexpensive activities like walking or running can totally avoid such costs.

Of course, such an economic evaluation is not completely about the money. Living a healthier lifestyle is an important trend of our days, as a typical answer to the faster growing sedentary industries and technologies where the humans are not active participants but simply spectators leading to a reduced physical activity.

From an economic point of view, the greatest benefits can be obtained by changing the behavior of the most sedentary persons out of a society - such a small step of moving from a totally sedentary life to some degree of physical activity actually represents a very important decision for life, while the savings can be an important motivator for these changes related to the physical activity patterns.

Regular physical exercises can be also seen as an investment intended to increase the personal health level for a better, longer, happier and more productive life having significant economic positive effects, so even from an economic point of view the physical activity increase for an active living is clearly a valuable investment for the future of any society. 
www.conferenceie.ase.ro

\section{Physical activity relative price for effective public health policies}

Physical activity can be also expressed in terms of money prices (participation fees, facility charges or even parking fares) and time expenses (travel time price). Of course, there is a strong negative correlation between the physical activity demand and the money and time prices per participation, i.e. high money prices and travel time are leading to a low degree of enrollment in physical activity.

Adjusting the relative price of (in)activity is an important instrument for public health policies. Thus, incentives related to the price of participation may lead to an increased amount of physical activity for the people already doing it. Also, tax reductions (road use, petrol or equipment fees) can be an effective tool for making the physical activity more accessible to the large public. Both approaches are effective not only for inactive persons but also for people not properly exercising.

Talking about regular goods or services, the market price itself is fair enough to express the amount of money needed for the acquisition. When speaking about physical activity, unlike usual commodities, not only different goods and services are required but also time is needed to properly practice, so a variety of prices are actually involved like participation fees (entrance, membership or facility charges), parking tickets, the cost of equipment or travelling time.

A utility (U) function maximization based on satisfaction can be used to explain the reason why some people do more physical exercises than others. The satisfaction is achieved from the consumption of physical activity and other goods/activities, all these being limited in terms of time and money (budget). The marginal rate of substitution (MRS) is the point in which the people are ready to give up some amount of goods/activities in exchange for physical activity, as shown in figure 3.

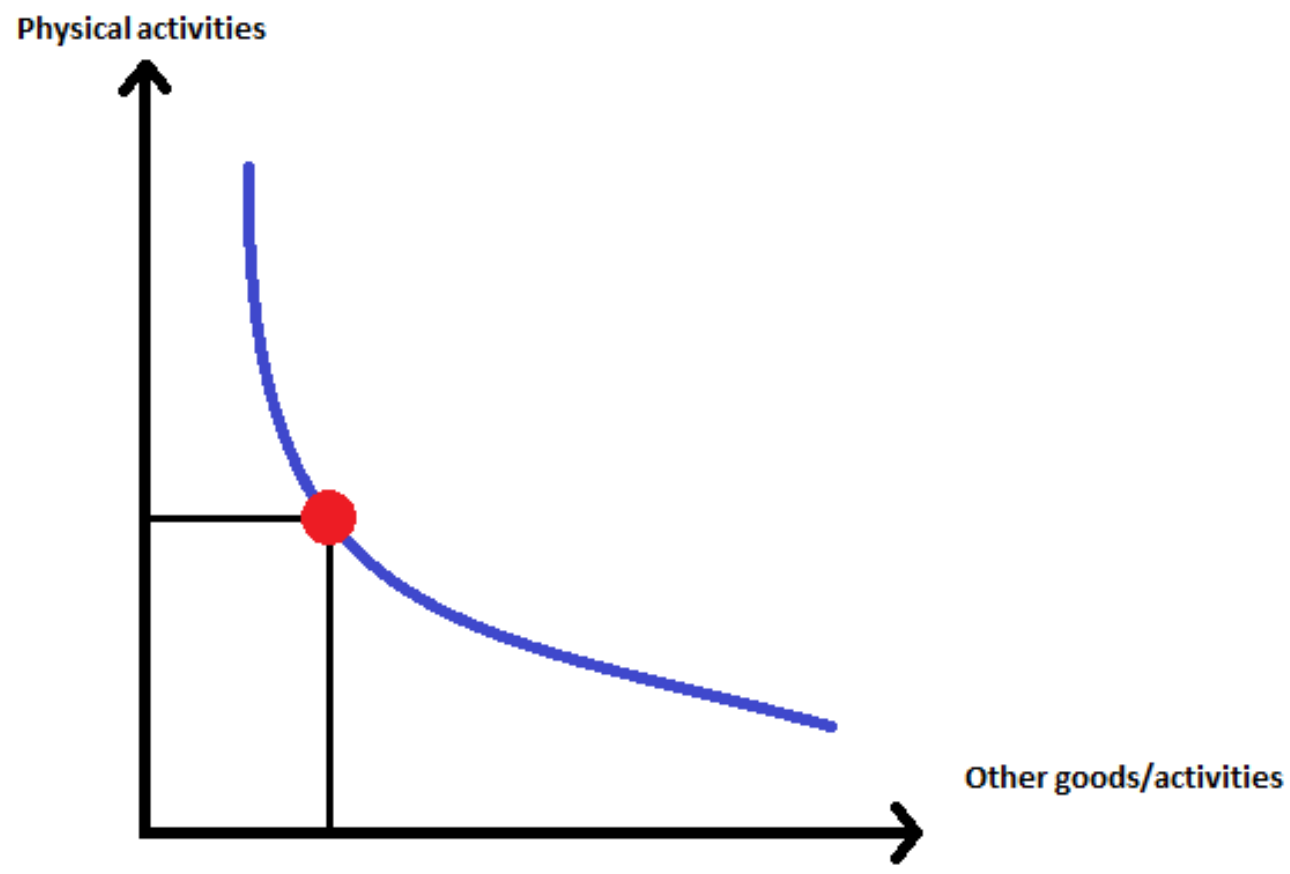

Fig. 3 Marginal rate of substitution involving physical activities

The utility is reaching the maximum level of satisfaction when $M R S$ equals the time and money prices for physical activity $(d A)$ and the other goods/activities $(d O G A)$, so the marginal rates of substitution (for physical activity and other goods/activities) are identical, as shown in equation 1. 


$$
M R S_{P A-O G A}=\frac{d P A}{d O G A}
$$

The behavioral changes intended to gain more individual control over the personal health, like empowering people to perform physical activities or simply accommodating the aspiration to do some workouts, can be promoted by different kind of incentives like subsiding the participation fee or tax reductions.

At this equilibrium level of consumption, by negative adjusting the relative price of (in)activity incentives, the time and money price of the physical activity will go down, meaning that any individual will actually obtain a greater utility by consuming one extra unit of physical activity compared to other goods, so to maintain this equilibrium state the individuals will need to consume more of physical activity, meaning really large benefits in terms of public health can be easily achieved by relatively small investments.

\section{Conclusions}

Mobile devices and applications can successfully bridge the divide between the healthful behaviors and sedentary patterns by shaping the user towards a "move more and sit less" approach able not to only add years to life but also life to the years [11].

The ever-increasing sensing capabilities of the smartphones of our days are able to reshape the daily people's life by activity recognition, so the technology can be a great physical activity enabler when properly used by setting the goals, monitoring the progress, reporting back the results and tracking overtime.

Behavioral changes are considered the main driver for the future public health improvements, so the mobile phones can be seen as important instruments for changing the human behavior for good.

A satisfaction based utility function maximization can be used to explain the reason why some people are performing more physical activity than others do.

By using incentives and subsidies to make the physical activity more accessible/affordable for the large public, the immediate effect of such economic measures is related to the people doing even more exercises than before with large benefits in terms of public health.

\section{References}

[1] ISCA/Cebr, The economic cost of physical inactivity in Europe, Internet: https://cebr.com/reports/the-costs-of-inactivity-in-europe/, June 2015

[2] F. Booth, C. Roberts, M. Laye, "Lack of Exercise Is a Major Cause of Chronic Diseases," Comprehensive Physiology, vol. 2, 2012, pp. 1143-1211

[3] World Health Organization, Physical activity. Internet: https://www.who.int/newsroom/fact-sheets/detail/physical-activity, February 23, 2018

[4] F. Alecu, P. Pocatilu, S. Capisizu, “An IoT Project for Vital Signs Monitoring," Informatica Economica, vol. 21, no. 2/2017, pp. 33-43

[5] S. Tarca, Fitness Trackers vs. Smartphone Apps, Internet: https://www.onedigital.com/blog/fitness-trackers-vs-smartphone-apps/

[6] M. Adamakis, "Comparing the Validity of a GPS Monitor and a Smartphone Application to Measure Physical Activity," Journal of Mobile Technology in Medicine, vol. 6, issue 2, pp. 28-38, august 2017.

[7] Activity Recognition API | Google Developers. Internet: https://developers.google.com/location-context/activity-recognition 
www.conferenceie.ase.ro

[8] Core Motion | Apple Developer Documentation. Internet: https://developer.apple.com/documentation/coremotion

[9] HealthKit | Apple Developer Documentation.

Internet:https://developer.apple.com/documentation/healthkit

[10] Google Fit SDK | Google Developers. Internet: https://developers.google.com/fit

[11] M. Drexler. "Adding years to life-and life to years," Internet: https://www.hsph.harvard.edu/news/magazine/centennial-years-to-life/. Fall 2013 